\title{
Voice based Electronic Mail System for Visually Challenged Individuals
}

\author{
Ajai Verma
}

\begin{abstract}
The improvement in available information systems managed to open numerous roads throughout the world for visually disordered people. Digital enviroment relying on audio input like this, the screen reviewer has aided blind individuals to greatly navigate online web based apps. But it is really challenging for blind individuals to reach all such contextual items, and even to use any internet facility. The progress of affordable internet-based technologies has widened up a number of possibilities for people who are blind worldwide. Screen readers have helped the blind to navigate internet apps enormously in an audio-representative virtual space, as we define a system architecture for voicemail that can be utilized readily and effectively by the blind individual to view emails. The output produced by this study has allowed blind individuals with a mobile phone device to submit and obtain voice-oriented emails in their mother tongue. Our graphical interface system was assessed against the conventional mail server graphical end user interface.
\end{abstract}

Keywords-Visually challenged people, IVR, Pronunciation to context converter, Mouse click event, Screen reader.

\section{INTRODUCTION}

In today's aera of communication, the online network performs a essentional role, human kind cannot even think a day without online commuincation. The web has opened the way for many options such as the worldwide attachment to personal press and advertising goes with that in association. It allow end users to interconnect with an amazing output over a wide range .Electronic mail is one of the prevalent and best communication technique. Electronic mails are regarded as the most secure manner of transmitting or getting significant data over the online network However some individuals in the globe today are unable to utilize the internet, while others are visually disabled who can't view things and therefore can't view device like computer, keyboard and alike, hence it is very hard for them to reside in this world of internet. As we know technology is enhancing it in every single minute and those enhanced technologies are available in the market in the form of products like screen readers, automated pronunciation recognition, context to pronunciation reader,Conversion of expression to writing but they're not so effective for them. A study demonstrates that over 460 million individuals worldwide have vision impaired individuals. That is, approximately 330 million individuals do not know how to use the web or email. Therefore, we have researched to make an email system based on voice for visually imparied indiviuals, that will assist people send their mail so that they could also communicate over internet through electronic mail. This architecture also reduces the

Revised Manuscript Received on 14 September, 2019.

Ajai Verma, Department of Computer Science and Engineering, Sanskriti University, Uttar Pradesh, India. (E-mail: sanpubip@gmail.com) intellectual load of the visually challenged indiviuals by using the keyboard to record and form letters.

\section{RELATED WORK}

The complete figure of e-mail accounts generated by 2014 is 4.1 billion, and by 2018 it is expected that 5.2 billion applications will be generated. This allows electronic messages the most frequently used communicating method. But most widely known mail services can not be used by visually impaired individuals in our day-to-day lives because the present electronic mail system does not provide any additional feature that could enable the end user to listen the written mail and other digital content presernt on the pannel of the computer device like laptop, mobile phone and alike. The visually impaired end user cannot visualize the context present on the panel; they cannot even think where to click to carry out the necessary operations. While many display end users are invented and accessible in market but still these individuals experience some small problems while using those products. One of the widely used product by visually impaired people is screen readers, they read any information present on the monitor and the individual must use shortcut keys to conduct operations and activities because the screen readers can not track the mouse position. While reviewing the working of this cited device i.e. Screen reader we came up with two things: one that person using the screen reader cannot use the mouse point because it is totally nuisance when the location of the pointer cannot be identified; the second, the person should be effectively acquainted with the use of the keyboard in which each button is placed. So we came with a conclusion that a person who is using this technology if that person is new to computer world can't use this service because that person does not know about the placement of keys in the keyboard or any other device similar to keypad. The complete figure of e-mail accounts generated by 2014 is 4.1 billion, and by 2018 it is expected that 5.2 billion applications will be generated. This allows electronic messages the most frequently used communicating method. But most widely known mail services cannot be used by visually impaired individuals in our day-to-day lives because the present electronic mail system does not provide any additional feature that could enable the end user to listen the written mail and other digital content present on the panel of the computer device like laptop, mobile phone and alike. The visually impaired end user cannot visualize the context present on the panel; they cannot even think where

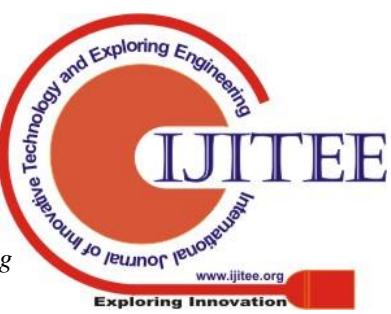


to click to carry out the necessary operations. While many display end users are invented and accessible in market but still these individuals experience some small problems while using those products. One of the widely used products by visually impaired people is screen readers, they read any information present on the monitor and the individual must use shortcut keys to conduct operations and activities because the screen readers cannot track the mouse position. While reviewing the working of this cited device i.e. Screen reader we came up with two things: one that person using the screen reader cannot use the mouse point because it is totally nuisance when the location of the pointer cannot be identified; the second, the person should be effectively acquainted with the use of the keyboard in which each button is placed. So we came with a conclusion that a person who is using this technology if that person is new to computer world can't use this service because that person does not know about the placement of keys in the keyboard or any other device similar to keypad. And also the screen readers read the context present on the screen in an ordered way, hence the person can understand only that context that is present in hyper context markup language format but the enhanced internet websites and web pages does not adopt this ideology to make their interface more end user friendly, eye catching thus it generate additional trouble for visual impaired end users.

\section{PROPOSED SYSTEM}

The proposed system has overcome all of the disadvantages listed above of the screen readers and other present system. The proposed system is a totally new concept based on mail systems. Access to information is the most significant thing considered in the development of the suggested system. In contrast to the present scheme that places more emphasis on the usability of ordinary consumers, our system relies more on all manner of individuals, even those with a visually impaired normal person, as well as those with illiteracy.

Interactive voice response animated pronunciation answer is the basis of the whole working of the proposed system. When using this scheme, the machine will prompt the end user to do certain activities and if the end user wants to enter the corresponding facilities, the proper procedure is requires to be carried out. The major benefit is that the customer will not need to use the keyboard. All activities are focused on occurrences with the button. The issue now is how blind consumers will discover the mouse pointer place. The system has not provided the end user, freedom to tap anywhere on the display because the specific place is not traceable by the blind person. The end user doesn't have to care about the placement of the mouse because interactive voice response will specify what kind of click is required to proceed further. And is available to all customers because it is focused on easy button taps and voice input, and screen shortcuts are not remembered. This system is available to all category of end user because it is focused on easy button taps and voice input, and screen shortcuts are not remembered. Also, those who do not study, need not be concerned because of interactive voice response facilities they can hear to the system's urging and execute their activities accordingly.

\section{DESIGN}

1. End user input module: Adobe Dreamweaver CS3 end user interface is intended. The entire page relies more on the effectiveness of comprehension interactive voice response rather than the appearance and touch of the system, as the system is created mainly for blind individuals for who glance and think are not as important as the effectiveness of knowing incentives.

2. Storage module: Our system retains an end user approval database and stores end user mails. A number of five charts are available. After much thought, the relation between them is allocated. Figure 1 shows the entity relation diagram of the entire scheme. All mail messages from the provider that is a specific consumer are stored in the inbox, in send-mail and in deposit schemes.

3. System Design: Figure. 2 show the entire layout of the system. It is an information stream diagram of level 2 that provides a comprehensive process stream of occurrences. As we can see, a mouse click occurrences are all conducted. Voice entry is also needed in some locations.

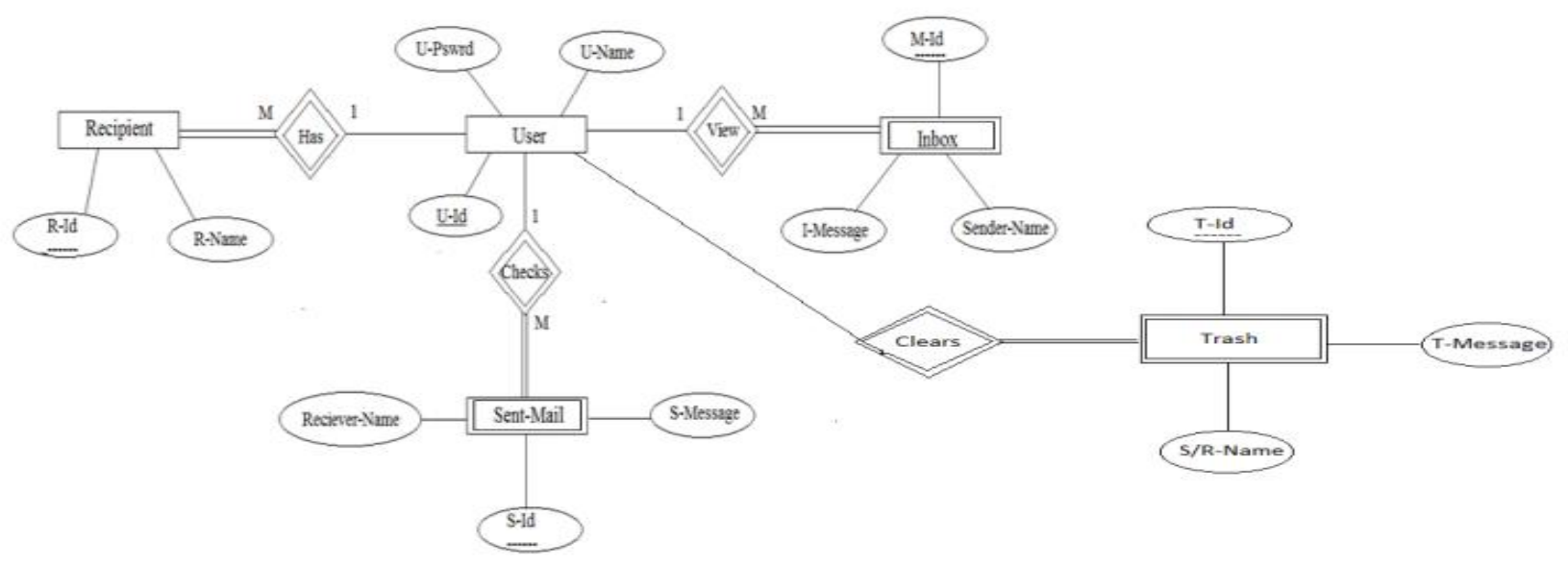

Figure 1 E-R Diagram of our system 


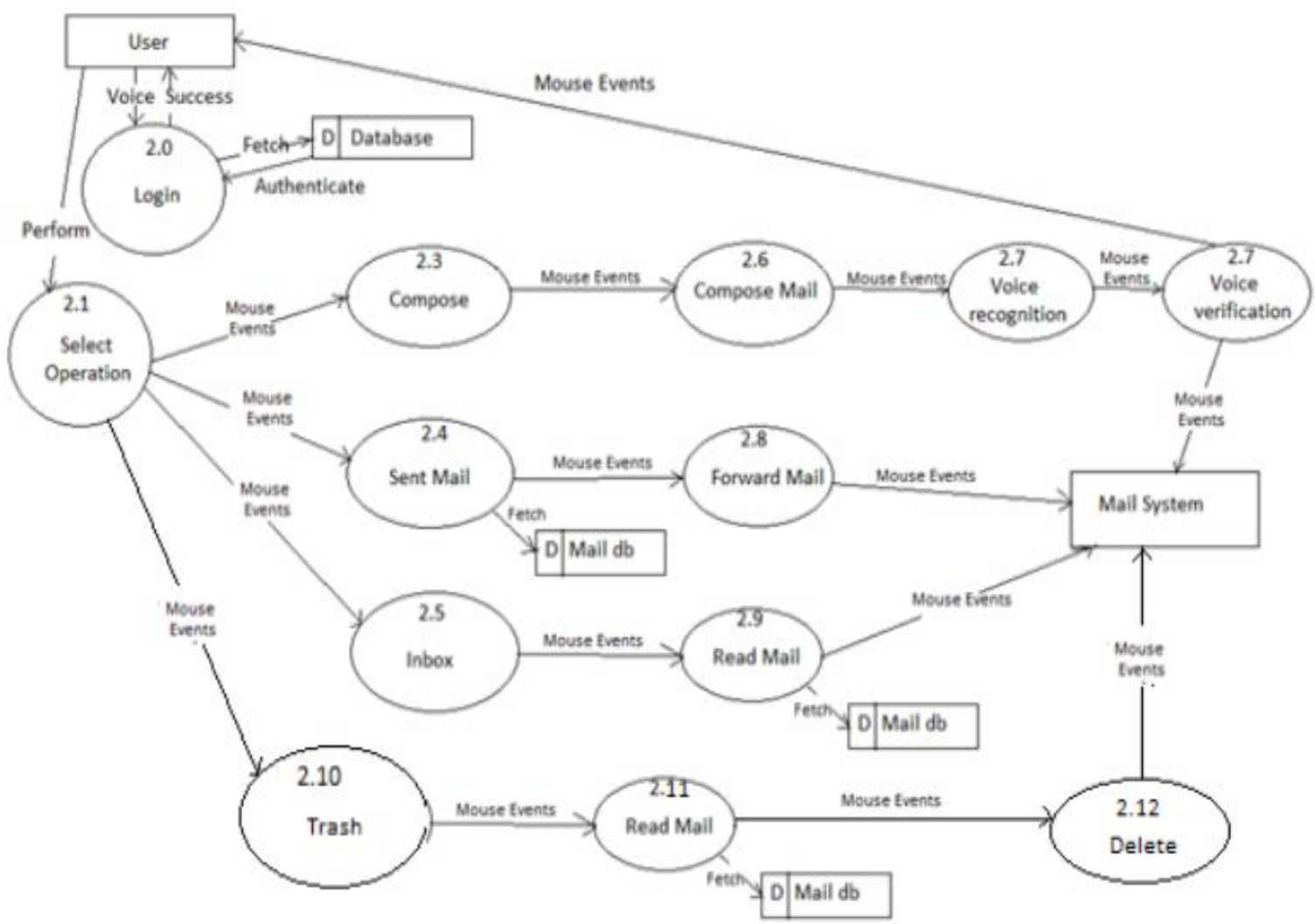

Figure 2 Level-2 Data Flow Diagram of our system

\section{RESULT}

This above described system has been developed by us. We have build the system in different types of modules working togther. All the modules have different functions.We have developed 7 modules for the sytsem namely:

1. Registration: The first module of the system is registration module.End user seeking to use the system must apply to acquire the code and end user name first. This module collects full end user data by telling the end user what type of data is required. The system confirm the details entered by end user by reading it alphabetically.

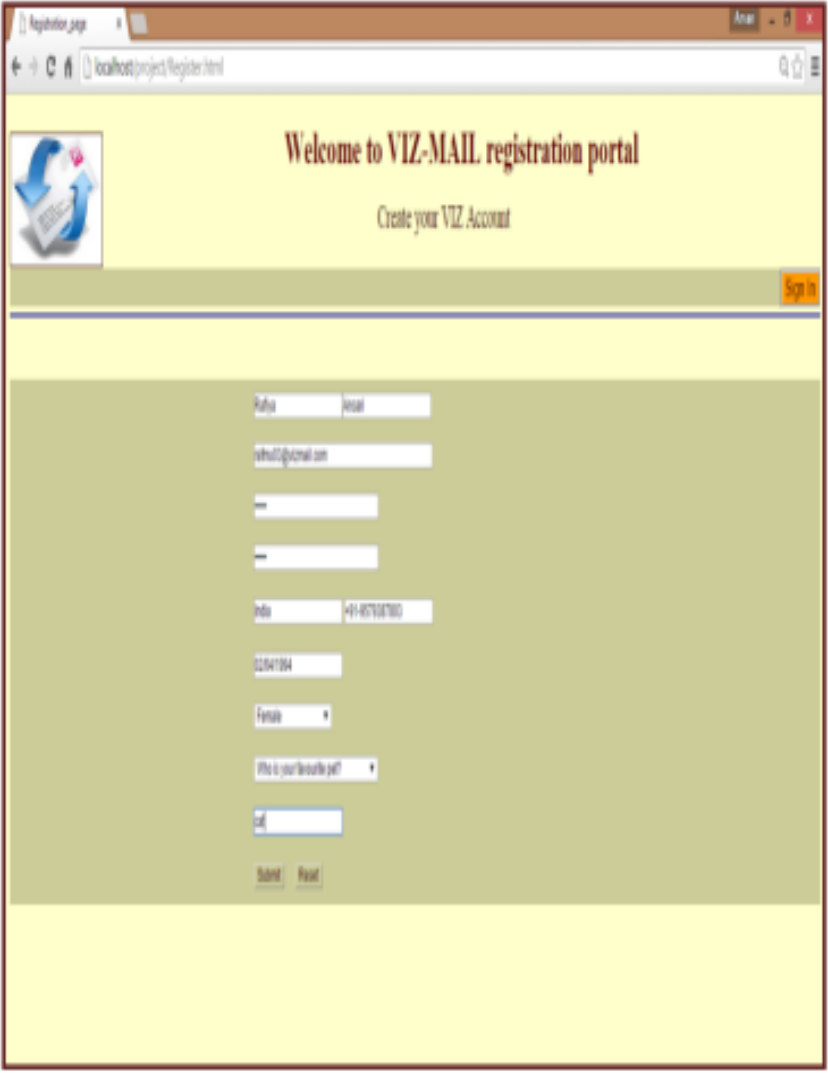

Figure 3 Registration Page

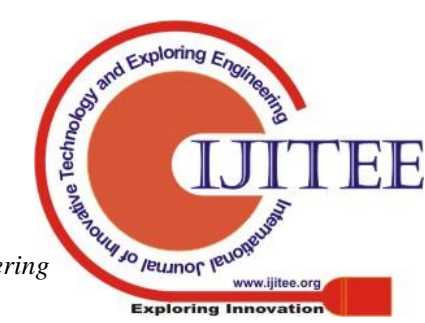


2. Login: Upon logging in the system, the end user will be capable to login. The end user needs to enter the end username and code in this package. This is recognized in words. Conversion of pronunciation to writing is performed and end users must check whether or not the particulars are registered properly, the input will be verified for getting access once the input is completed properly and the end user is permit to visit the website.

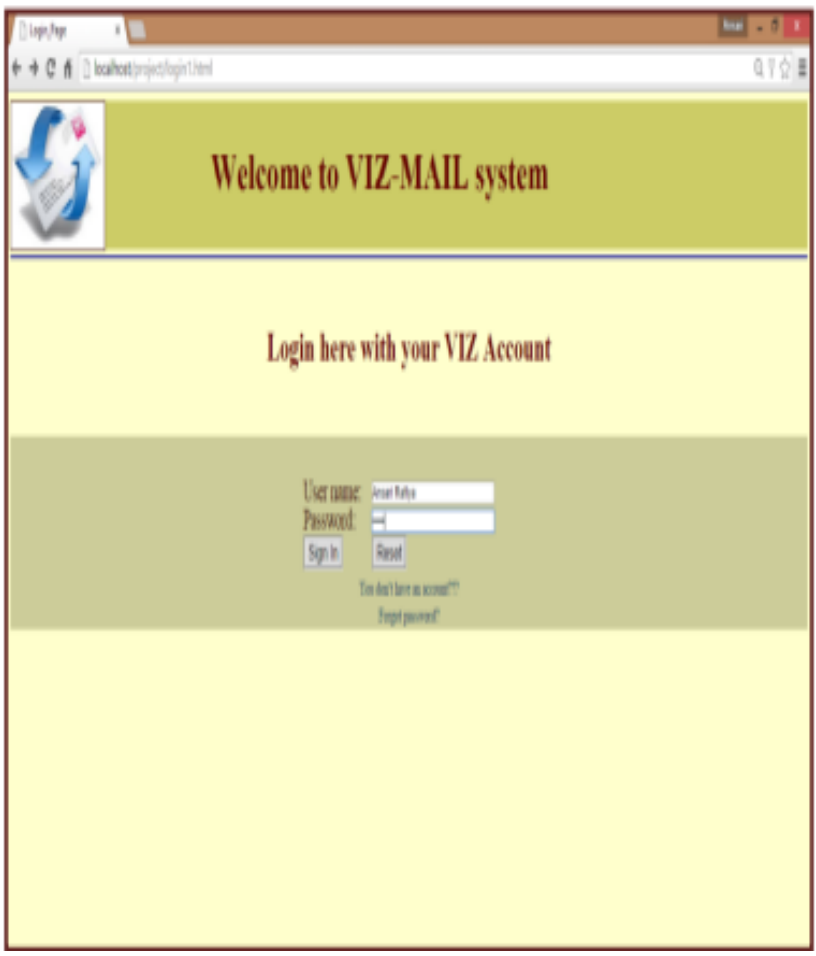

Figure 4 Login Page

3. Password Recovery: If the authorized user forgets the code word and cannot log on, he / she can choose the forgotten part of password. The operator is instructed to register username first in this module. The security question is checked in the repository depending on the username. This was the issue that was raised during registration.
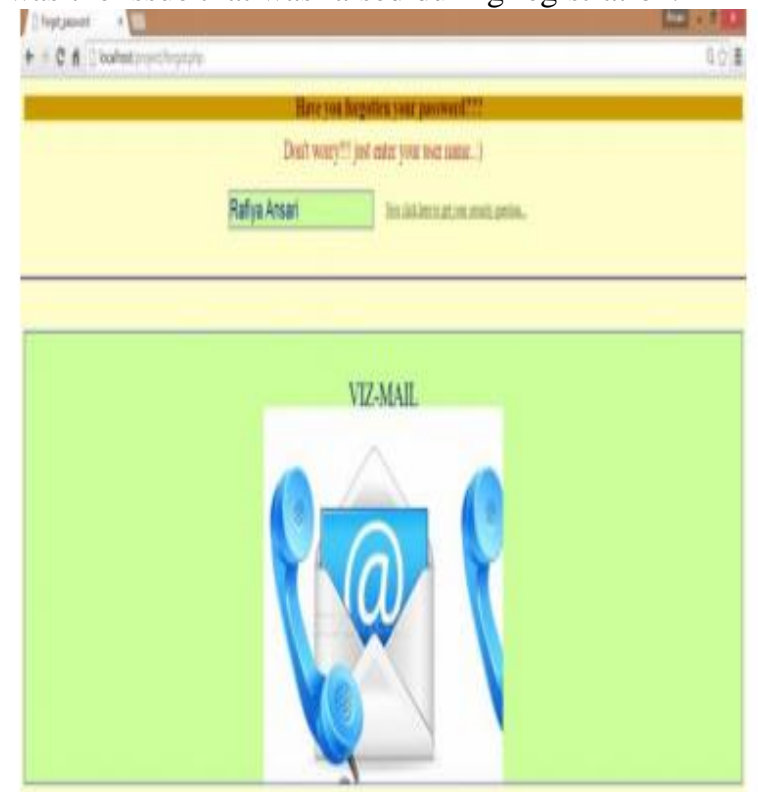

Figure 5 Forgot Password
4. Initial page: Once logged in effectively, the client is redirected to this section. The customer can now conduct activities that the customer wants to carry out from this section. Provocation will provide the necessary service with a mouse button procedure. The double-click occurrence is specifically intended for the end user to sign out of the system. This is stipulated at the start of log-in by the timely.

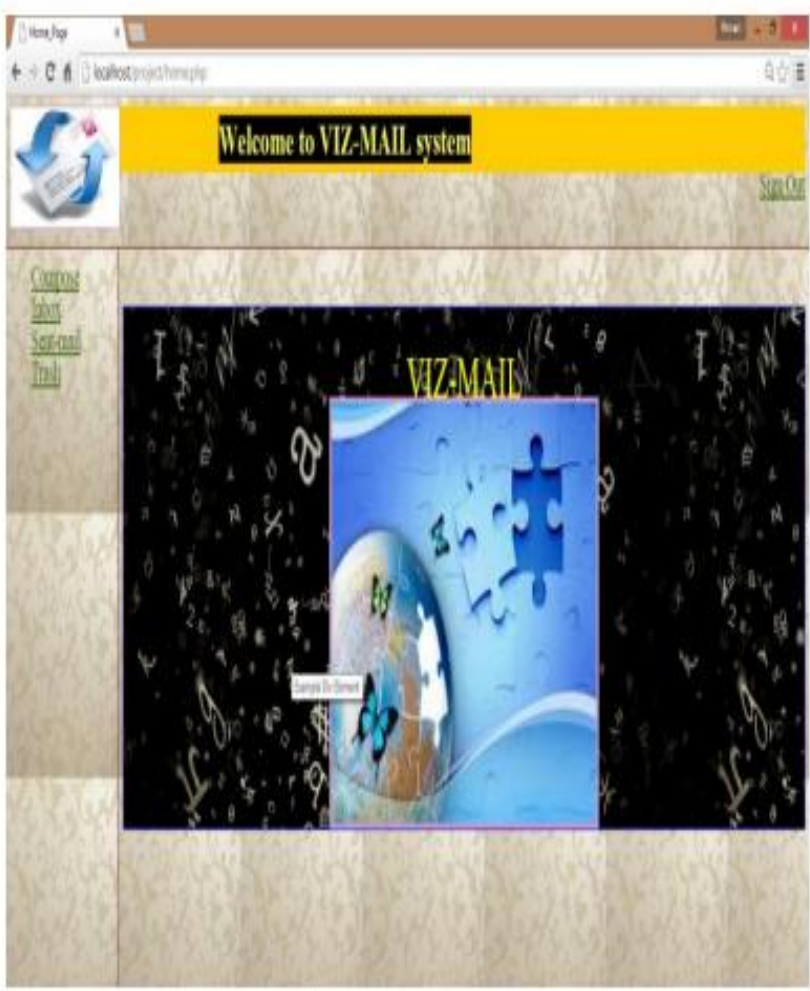

Figure 6 Home Page

\section{CONCLUSIONS}

In this paper we have proposed a system that helps individuals with visual impairments to effectively obtain access to electronic mail. This proposed system helps them to overcome certain disadvantages that blind individuals have had to face before they use electronic mails. Any end end user of any age category may use this email system easily. It also has the function of pronunciation-to-context and pronunciation-to-pronunciation with a pronunciation viewer, making both the optically impaired and slightless person responsible for designing a system..

\section{REFERENCES}

1. T.shabana, a.anam, a.rafiya3, k.aisha, "voice based email system

forblinds"http://www.ijarcce.com/upload/2015/january/ij arcce5c.pdf

2. Code project, "pronunciation recognition" http://www.codeproject.com/articles/5820/pronunciationr ecognition [3] Ummuhanysifa u., nizar banu p k, "voice based search engine and web page reader". In international journal of computational engineering research (ijcer).

3. Http://www.ijceronline.com/papers/special\%20issue/a01 05.pdf 
4. Arjun aj, "voice based email for blinds", slide share http://www.slideshare.net/123arjun1 /voicebased-emailfor-blinds

5. C. Kang, H. Jo and B. Kim, "A Machine-to-Machine based Intelligent Walking Assistance System for Visually Impaired Person", The Journal of KICS, vol. 36, no. 3, (2011), pp. 195-304.

6. S. Kumar, M. A. Qadeer and A. Aupta, "Location Based Service using Android", Internet Multimedia Service Architecture and Applications, IEEE International Conference, (2009).

7. H. -W. Jung, "Smartphones and future changes", The Korea Contents Association, vol. 8, no. 2, (2010).

8. I -H. O, J. S. Bae, D. -W. Park and Y. -H. Sohn, "Implementation of Location Based Service(LBS) using GPS for Various Sizes of Maps", Korean Institute of Information Technology, vol. 8, no. 4, (2010).

9. G. E. Lee and J. W. Lee, "Google Android phone Personal open market", Korean Multimedia Society, Fall Conference, (2009), pp.346-349. 